\title{
Women's perceptions and experiences of nutritional advice and support during their climacteric
}

During climacteric, women's bodies move from a reproductive to non-reproductive state, subsequent to declining oestrogen levels ${ }^{(1)}$. Menopause occurs on average at 51 years for UK-women ${ }^{(1)}$ while average female life expectancy is 83.1 years ${ }^{(2)}$. Therefore, women potentially spend around 32 years in an oestrogen-depleted state, associated with increased health vulnerability and elevated cardiovascular disease or osteoporosis risk ${ }^{(1)}$. These conditions could be improved with dietary modification, although how nutrition advice is communicated is unclear. The study aimed to explore women's understanding of climacteric and experience in accessing nutrition information and advice.

Twenty women in climacteric, aged 40-60, participated in 3 single-category focus groups. Recruitment used purposive snowball sampling via social media. Focus groups were conducted through video conferencing using a topic guide. Post-session, transcripts were anonymised, transcribed verbatim and analysed using phenomenological thematic analysis. Applying Braun and Clarke's process $^{(3)}$, themes were derived at semantic level using realist theoretical analysis. Data trustworthiness was established, utilising Lincoln and Guba's framework $(1985)^{(4)}$.

Sociodemographic data revealed participants were predominantly white $(95 \%)$. Cohort's mean age was 49 with BMI average of $24.71 \mathrm{~kg} / \mathrm{m} 2$. The majority $(75 \%)$ were non-smokers with $25 \%$ stating being ex-smokers. Thematic analysis indicated limited support in relation to identifying and managing menopausal symptoms; many struggled acknowledging them. Sleep issues were common, affecting cognitions and wellbeing. Additionally, perception of menopause as indicative of ageing was lacking. Clinical support was often perceived negatively, some indicating it a "postcode lottery". Only some reported positive experiences, markedly from empathetic female GPs. Others felt GP's lacked knowledge, determining them as "too young", subsequently not returning, highlighting missed opportunities for screening/intervention. A second theme identified motivating factors driving change during climacteric. Several participants indicated changing dietary behaviour in response to weight or appetite change, or stabilising mood. Participants expressed concerns over increased vulnerability to injury with many suggesting a desire to age healthily, maintaining independence and mobility. However, there was limited knowledge of potential health conditions (e.g., cardiovascular disease) post menopause despite majority indicating self-research. Interestingly, participants indicated no association between nutrition and menopause "I haven't correlated menopause, midlife and what I eat" while commercial diet plans were often perceived as nutritional knowledge sources. Peers, including friends and family, were frequently acknowledged sources of information alongside social media groups. Importantly, in the current study no participants had received direct nutrition information relating to menopause transition noting "Nutrition is a bit of a black spot". However, there was significant desire for information enabling informed choices about health needs.

Overall, the study indicates paucity of knowledge regarding menopause, long-term menopause sequalae or nutrition during climacteric period. Findings suggest a need for high quality resources, supporting women in improving long-term quality of life.

\section{References}

1. Dunneram Y, Greenwood DC \& Cade JE (2021) Maturitas 143, 165-170.

2. Leon DA, Jdanov DA \& Shkolnikov VM (2019) Lancet 4, e575-582.

3. Braun V \& Clarke V (2006) Qual Res Psychol 3, 77-101.

4. Nowell LS, Norris JM, White, DE, et al. (2017) Int J Qual Methods 16, 1-13. 